\title{
Effect of long-acting testosterone undecanoate treatment on quality of life in men with testosterone deficiency syndrome: a double blind randomized controlled trial
}

\author{
Seng-Fah Tong ${ }^{1}$, Chirk-Jenn $\mathrm{Ng}^{2}$, Boon-Cheok Lee ${ }^{3}$, Verna-KM Lee ${ }^{4}$,Ee-Ming Khoo ${ }^{2}$, Eng-Giap Lee ${ }^{2}$ \\ and Hui-Meng Tan ${ }^{2,3}$
}

This study aimed to investigate the effect of intramuscular injection of testosterone undecanoate on overall quality of life (QoL) in men with testosterone deficiency syndrome (TDS). A randomized controlled trial over a 12-month period was carried out in 2009. One hundred and twenty men aged 40 years and above with a diagnosis of TDS (serum total testosterone $<12 \mathrm{nmol} \mathrm{I}^{-1}$ and total Aging Male Symptom (AMS) scores $\geqslant 27$ ) were invited to participate. Interventions comprised intramuscular injection of either placebo or $1000 \mathrm{mg}$ testosterone undecanoate, given at weeks $0,6,18,30$ and 42 . This paper presents the secondary analysis of QoL changes measured in the scores of Short-Form-12 (SF-12) scale at baseline, weeks 30 and 48 after the first injection. A total of 56/60 and 58/60 men from the active treatment and placebo group, respectively, completed the study. At week 48 , before adjusting for baseline differences, the QoL of men in the treatment group improved significantly in five out of the eight domains on SF-12. The physical health composite scores improved 4.0 points from a baseline of $41.9 \pm 7.0$ in the treatment group compared to 0.8 point from a baseline of $43.7 \pm 7.1$ in the placebo group $(F=3.652, P=0.027)$. The mental health composite scores improved 4.4 points from a baseline of $37.1 \pm 9.0$ in the treatment group compared to 1.0 points from a baseline of $37.6 \pm 7.9$ in the placebo group $(F=4.514, P=0.018)$. After adjusting for baseline differences, significant improvement was observed in mental health composite scores, but not in physical health composite scores. Long-acting testosterone undecanoate significantly improved the mental health component of QoL in men with TDS.

Asian Journal of Andrology (2012) 14, 604-611; doi:10.1038/aja.2011.178; published online 28 May 2012

Keywords: hypogonadism; randomized controlled trial; quality of life; testosterone deficiency syndrome (TDS); testosterone therapy

\section{INTRODUCTION}

Testosterone deficiency syndrome (TDS) is increasingly being recognized as a significant health problem in aging men. ${ }^{1-7}$ In the United States, the estimated prevalence of symptomatic androgen deficiency in men aged $30-79$ years is $5.6 \% .^{8}$ In Malaysia, the estimated prevalence of TDS, defined as serum total testosterone (TT) level $\leqslant 11 \mathrm{nmol} 1^{-1}$ and positive score on the Androgen Deficiency in the Aging Male questionnaire, in men aged 40 years and above, is $6 \%{ }^{9}$

The adverse impacts of TDS include (i) physical impairment, such as loss of physical strength, loss of muscle mass, increased visceral fat leading to a higher risk for metabolic syndrome and premature death; (ii) decreased sexual function, such as loss of secondary sexual characteristics, decreased libido and erectile dysfunction; and (iii) psychological disturbances such as mood changes and sleep disturbance. ${ }^{2,10}$ As a result, the quality of life (QoL) in men with TDS is affected, as shown in studies using various disease-specific (St Louis University Androgen Deficiency in Aging Males, Aging Male Symptom (AMS), Sickness Impact Profile) and generic (SF-36, Psychological General Well-Being, Health-related QoL) QoL scales. ${ }^{11-16}$ Therefore, treatment is recommended for men with TDS who are symptomatic.
Current options of testosterone replacement therapy include oral therapies, topical creams, transdermal patches, subdermal implants and intramuscular injections. ${ }^{1,2-17}$ Intramuscular injection of long acting testosterone undecanoate (TU) has an advantage of providing a stable and physiological level of testosterone. It is injected four times a year, which renders it an attractive option. ${ }^{17,18}$

However, most studies have found benefits of testosterone replacement therapy on physical factors; its effect on patient-reported QoL remains uncertain, ${ }^{2}$ particularly its effect on the overall QoL of men with TDS. ${ }^{19}$ The aim of this paper, therefore, is to investigate the effect of intramuscular injection of TU on the overall health-related QoL in men with TDS over a 12-month period. The findings in this paper are part of a larger project investigating the effect of intramuscular injection of TU on men with TDS.

\section{MATERIALS AND METHODS}

Study design

It was a double-blind, parallel, randomized, placebo-controlled trial with an allocation ratio of $1: 1$. The study was approved by the Medical Ethics Committee, University of Malaya Medical Centre (approval

\footnotetext{
${ }^{1}$ Universiti Kebangsaan Malaysia, Kuala Lumpur 56000, Malaysia; ${ }^{2}$ University of Malaya, Lumpur 56000, Malaysia; ${ }^{3}$ Sime Darby Medical Centre, Selangor 47500, Malaysia and ${ }^{4}$ International Medical University, Kuala Lumpur 57000, Malaysia

Correspondence: Dr S-F Tong (sengfahtong@gmail.com)

Received: 23 July 2011; Revised: 11 September 2011; Accepted: 27 November 2011; Published online: 28 May 2012
} 
number: 631.11). The study was not registered in the trial registry as at the time of conduct of the study, it was not a requirement to do so under the Malaysian Trial registry at the commencement of the study. The primary end point of the main study was treatment effects on the AMS scores, and the finding is published elsewhere. ${ }^{20}$ In this paper, we report the treatment effects on the eight domains of the Medical Outcomes Study Short-Form-12 (SF-12) by Quality Metric Incorporated, Lincoln, a generic health-related QoL questionnaire.

\section{Participants}

The participants were recruited by telephone invitation from a cohort of randomly selected men aged 40 years and above in an urban community. The cohort was the participants in the 2008 Subang Aging Male project, ${ }^{21}$ who were randomly selected using the electoral roll. Those who had a low serum testosterone level in the survey were contacted. The participants were explained about the study and consent was obtained before the screening assessment was made. They were reimbursed MYR50.00 (USD16.00) every visit only for travel expenses and they were not financially coerced to be in the trial. The participants who consented were first screened for TDS with an early morning TT test and AMS questionnaire. If the TT was $<12 \mathrm{nmol}^{-1}$ and the total AMS scores were $\geqslant 27$ (corresponding to mild to severe symptoms of TDS), the participants were recalled for a formal screening visit. Although AMS scores of $\geqslant 27$ were non-specific, it is a useful tool to identify participants with testosterone deficiency. ${ }^{22}$ The diagnosis of TDS was confirmed subsequently with early morning $\mathrm{TT}<12 \mathrm{nmol}^{-1}$ on two occasions. The aim of the formal screening visit was to establish if the participant fulfilled the inclusion criteria that included: age 40-70 years old; had at least 'mild' symptoms for all three AMS subscales (scores of $\geqslant 9$ in somatovegetative; $\geqslant 6$ in psychological; and $\geqslant 6$ in sexual subscales) or a total AMS scores $\geqslant 27$; early morning TT $<12 \mathrm{nmol} \mathrm{l}^{-1}$ on two occasions; and prostate-specific antigen (PSA) $<4 \mathrm{ng} \mathrm{ml}^{-1}$. The exclusion criteria were: uncontrolled diabetes mellitus (HbA1c $>8 \%$; because it may confound the symptoms of TDS), clinical hypothyroidism or hyperthyroidism, haematocrit $>55 \%$, known case of prostate cancer, androgen dependent carcinoma of the male mammary gland, past or present liver tumours, and other significant medical conditions (American Society of Anesthetists score $>3$ ) or psychological conditions (psychotic, schizophrenic or manic depression) or clinically diagnosed sleep apnea. Participants were also excluded if they were on medications known to interfere with testosterone metabolism, hypersensitive to the active substance or excipients of the study medication, on testosterone treatment (transdermal, oral or intramuscular) within the previous 6 months or testosterone implants within the previous 12 months. These criteria were agreed consensually by a team of local experts.

\section{Sample size}

The sample size was calculated based on the improvement in AMS score, the primary end point of the project. With a two-sided alpha value of 0.05 and power of $80 \%$, a sample size of 60 subjects per group is required to detect a 4.64 point reduction in the AMS scores from the baseline (30.8) at 48 weeks after intervention with a population standard deviation of $8.98 .^{23}$ In addition, this sample size is able to detect a minimum of 8.78 point difference in QoL scores from baseline with an estimated population standard deviation of $17 .^{24}$

\section{Study protocol}

The study was conducted at a tertiary medical centre in Malaysia. All participants who fulfilled the study criteria were assessed by the clinical investigators to establish their baseline profiles, which included: weight, height, blood pressure and prostate status by digital rectal examination; current medications and concurrent illnesses; biochemistry profile such as TT, haematocrit, liver function test aspartate transaminase, alanine transaminase, albumin, bilirubin, gamma-GT and serum PSA; and International Prostate Symptom Score (IPSS) and SF-12 questionnaires.

At visit 1, the eligible participants received an intervention package indexed with a serial number that contained five vials of either placebos or active medication, prepared in an identical form. Each serial number with its code for either placebo or active medication was kept in a sealed envelope. The supplier of the intervention packages was not involved in any stage of the trial. The participants, clinical investigators and trial site manager were blinded to the intervention. Revealing the codes within the trial period was permitted only when there was an occurrence of serious adverse events, which occurred in four participants, and subsequently they were discontinued from the trial.

At weeks 6, 18, 30, 42 and 48 after the first intervention, repeat physical assessments (except digital rectal examination) were done. Serum TT and biochemistry profile were repeated at weeks 18 and 48; digital rectal examination, IPSS and SF-12 questionnaires were repeated at weeks 30 and 48 . The serum TT levels were not revealed to participants or investigator until the trial period was over. All laboratory investigations were performed by a single laboratory to maintain standardisation of measurements and reference points.

\section{Intervention}

All participants received five injections from the package allocated to them at weeks $0,6,18,30$ and 42 . The active treatment was $1000 \mathrm{mg}$ of testosterone undecanoate in $4 \mathrm{ml}$ of castor oil, while the placebo was castor oil of the same volume and appearance. The injection was given as slow bolus intramuscularly at the gluteal region over $1 \mathrm{~min}$.

\section{Outcome measurement tool}

This paper reports the scores of Medical Outcomes Study SF-12. ${ }^{25}$ SF-12 is a self-administered validated tool to measure generic health-related QoL. It has an excellent correlation with SF-36, ${ }^{25-28}$ which is a longer version and the predecessor of SF-12. The excellent correlation between SF-36 and SF-12 was confirmed in Asian setting. ${ }^{28}$ The validity of the Malay version SF-36 has been established in the Malaysian population. ${ }^{24}$ SF-12 was selected because of its validity and brevity. It measures eight domains of QoL (physical functioning, physical role functioning, bodily pain, general health, vitality, social functioning, emotional role functioning and mental health) and has two composite scores for physical and mental health. Higher scores on SF-12 indicate better QoL. The participants completed the SF-12 questionnaire at baseline, weeks 30 and 48 after the intervention.

\section{Safety monitoring and withdrawal}

Participants' safety was monitored by scheduled assessment and selfreporting of adverse events. Scheduled assessment included a clinical assessment at every visit (with a repeat digital rectal examination at week 30) and monitoring of biochemistry profile at weeks 18 and 48 . Every participant was asked to report any symptom after commencement of the intervention. A mandatory review and consideration for withdrawal from the study would be undertaken if any one of the following criteria was noted: a rise in PSA of $\geqslant 50 \%$ from baseline or above $4 \mathrm{ng} \mathrm{ml}^{-1}$; clinical abnormality on digital rectal exam; a significant worsening of IPSS score; haematocrit $>55 \%$; or any report 
of adverse significant events. The PSA cutoff level of $4 \mathrm{ng} \mathrm{ml}^{-1}$ was used because study showed the prevalence of prostate cancer in Asian men was very low when the PSA was $\leqslant 4 \mathrm{ng} \mathrm{ml}^{-1} .^{29}$

\section{Statistical analyses}

The data analysis was done using the Statistical Package for the Social Sciences (SPSS Inc., Chicago IL, USA) version 15. Descriptive statistics were used for baseline demography, outcome measurements and adverse events. Exact contingency table method was used for categorical data to compare baseline profile and adverse events between active treatment and placebo group, while independent sample $t$-test was used for continuous data. The responses to outcome measures for each group were calculated based on the difference from their baseline values. The effects of active treatment on QoL scores were estimated using repeated measure ANOVA by including the interaction terms of 'intervention' $X$ 'time'. The two-sided level of significance $(P)$ was set at 0.05 .

\section{RESULTS}

A total of 504 men were contacted and 284 men presented to the study centre for assessment of eligibility (Figure 1). The remainders of 220 men were uninterested. Sixty participants were recruited for each group of the interventions. In the active treatment group, $93.3 \%$ (56/60) completed the trial and in the placebo group, $96.7 \%(58 / 60)$ completed the trial. Six participants did not complete the study. Two participants from the active treatment group withdrew because of relocation; two participants were admitted for chest pain-one in each group after visits 4 and 5, respectively, who were diagnosed to have ischaemic heart disease; and the other two participants, one from each group, had died of acute myocardial infarction after visit 5 .

The baseline characteristics for both groups were comparable except for metabolic parameters and AMS scores (Table 1). The body mass index, waist circumference and diastolic blood pressure were
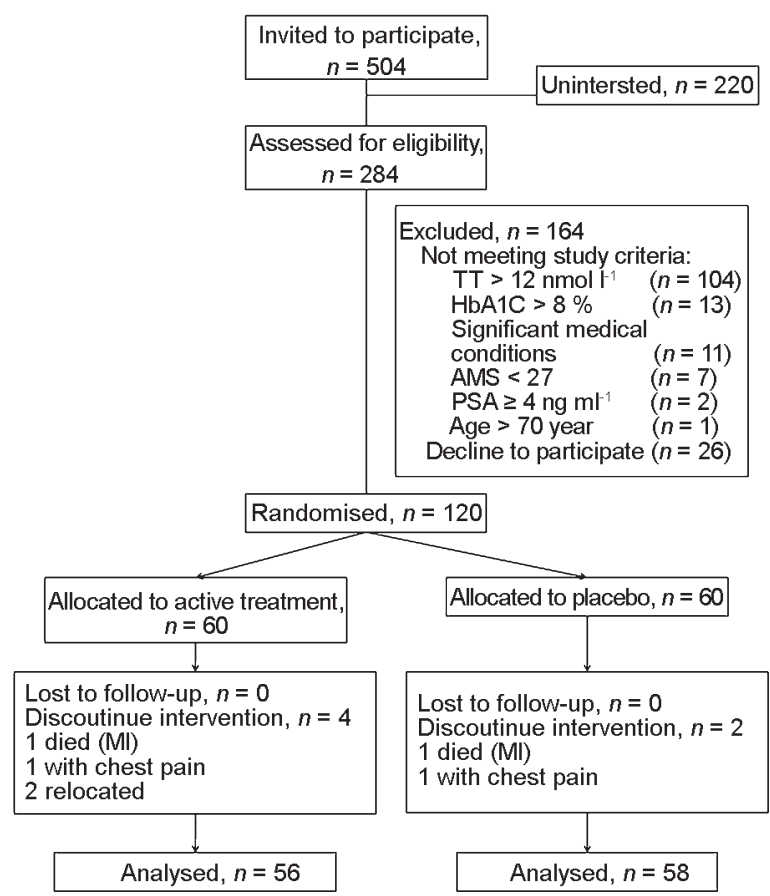

Figure 1 Progress of participants involvement in the study. AMS, Aging Male Symptom; PSA, prostate-specific antigen. MI, myocardial infarction; TT, total testosterone.
Table 1 Baseline characteristics of the two groups

\begin{tabular}{|c|c|c|c|}
\hline & Placebo & Active treatment & $\mathrm{P}$ \\
\hline Age $^{\text {a }}$ (year) & $53.0 \pm 8.2$ & $53.4 \pm 7.5$ & 0.771 \\
\hline Body mass index ${ }^{\mathrm{a}}\left(\mathrm{kg} \mathrm{m}^{-2}\right)$ & $28.2 \pm 4.5$ & $30.4 \pm 5.2$ & 0.017 \\
\hline Waist circumstances ${ }^{a}(\mathrm{~cm})$ & $96.3 \pm 10.7$ & $102.7 \pm 12.3$ & 0.003 \\
\hline Systolic blood pressure $(\mathrm{mmHg})$ & $127.9 \pm 10.4$ & $132.5 \pm 14.6$ & 0.053 \\
\hline Diastolic blood pressure ${ }^{\mathrm{a}}(\mathrm{mmHg})$ & $81.4 \pm 6.4$ & $84.1 \pm 7.4$ & 0.031 \\
\hline Total testosterone $\mathrm{a}^{\mathrm{a}}\left(\mathrm{nmol} \mathrm{I}^{-1}\right)$ & $9.1 \pm 1.8$ & $8.9 \pm 2.0$ & 0.546 \\
\hline $\operatorname{SHBG}^{\mathrm{a}}\left(\mathrm{nmol} \mathrm{I}^{-1}\right)$ & $20.9 \pm 6.9$ & $21.6 \pm 7.0$ & 0.567 \\
\hline Total AMS score ${ }^{a}$ & $38.2 \pm 11.9$ & $42.9 \pm 13.2$ & 0.043 \\
\hline Somatic subscale & $15.8 \pm 5.4$ & $17.6 \pm 5.6$ & 0.078 \\
\hline Psychological subscale & $10.0 \pm 4.0$ & $11.6 \pm 4.5$ & 0.045 \\
\hline Sexual subscale & $12.4 \pm 4.3$ & $13.7 \pm 4.4$ & 0.092 \\
\hline \multicolumn{4}{|l|}{ Ethnicity ${ }^{b}$} \\
\hline Malay & $15(25.0)$ & $16(26.7)$ & 0.972 \\
\hline Chinese & $25(41.7)$ & $25(41.7)$ & \\
\hline Indian & $17(28.3)$ & $17(28.3)$ & \\
\hline Others & $3(5.0)$ & $2(3.3)$ & \\
\hline \multicolumn{4}{|l|}{ Comorbidities $^{\mathrm{b}}$} \\
\hline Hypertension & $20(33.3)$ & $29(48.3)$ & 0.095 \\
\hline Diabetes mellitus & $9(15.0)$ & $14(23.3)$ & 0.246 \\
\hline Dyslipidemia & 20 (33.3) & $22(36.7)$ & 0.702 \\
\hline Coronary artery disease & $3(5.0)$ & $6(10.0)$ & 0.298 \\
\hline
\end{tabular}

Abbreviations: AMS, Aging Male Symptoms; SHBG, serum hormone-binding globulin. ${ }^{a}$ Data expressed as mean \pm s.d.

${ }^{\mathrm{b}}$ Data expressed as $n(\%)$.

significantly lower in the placebo group than the active treatment group. The total AMS and psychological subscale scores were higher in the active treatment group.

\section{Serum total testosterone response to intervention}

Serum TT levels increased significantly in both placebo and active treatment groups. In the placebo group, mean TT slightly increased from 9.1 at baseline to $11.2 \mathrm{nmol}^{-1}$ at week $48 \quad(F=12.527$, $P<0.001$ ), whereas in the active treatment group, mean TT significantly increased from 8.9 at baseline to $23.7 \mathrm{nmol}^{-1}$ at week $48(F=181.273$, $P<0.001)$. The significant increase in the placebo group occurred within the first 18 weeks (TT=11.4 nmol $1^{-1}, F=12.527, P<0.001$ ) and it reached a plateau thereafter; however, the levels remained below $12 \mathrm{nmol}^{-1}$. On the other hand, mean TT in the active treatment group raised to the normal range $\left(>12 \mathrm{nmol} \mathrm{l}^{-1}\right)$ after week 18 and continued to increase up to week 48 . The increase in active treatment group over 18 and 48 weeks is significant compared to placebo group $(F=62.001$, $P<0.001$ ) (Figure 2).

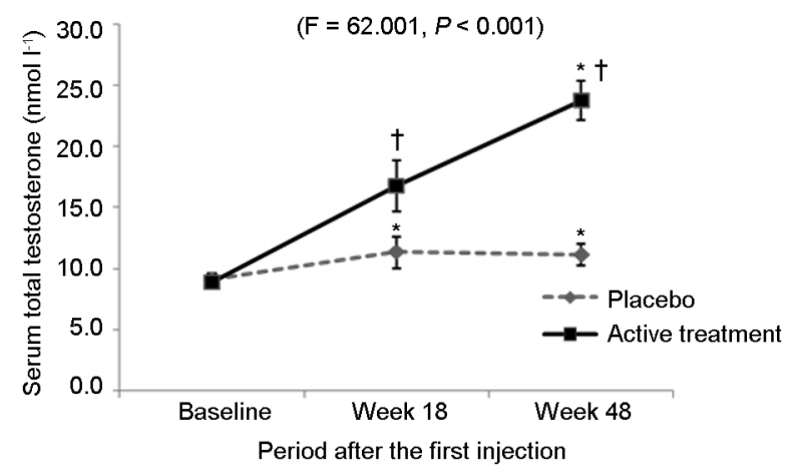

Figure 2 Mean serum total testosterone (with 95\% CI) level during the trial period. $* P<0.001$, compared to baseline; $\uparrow P<0.001$, compare to placebo group. 


\section{Changes in QoL}

SF-12 scores improved significantly in all domains of QoL from baseline in the active treatment group, whereas only the general health domain showed significant improvement in the placebo group (Table 2). Repeated measures of ANOVA comparing the effects of active treatment and placebo on QoL scores over time showed improvement in most domains in the active treatment group (Table 2). The most significant improvement in the SF-12 scores was seen in vitality $(+7.0)$, followed by general health $(+6.1)$, social functioning $(+5.7)$, physical role functioning $(+3.0)$ and emotional role functioning $(+1.8)$. The physical and mental health composite scores were significantly improved in the active treatment group but there was no difference in the placebo group. There was a significant difference in the physical and mental health composite scores between the active treatment and placebo groups.

The mean changes in QoL scores from baseline to week 48 ranged from +1.9 to +7.0 in the active treatment group compared to -0.2 to +2.3 in the placebo group. In the active treatment group, significant improvement $(P<0.05)$ was seen as early as week 30 for all domains of QoL except for physical functioning (Figure 3). At week 48, general health, physical functioning and role functioning improved further $(P<0.05)$ (Figure $3 \mathbf{b})$, but no further significant improvement was seen in the other domains (Figure 3a). In the placebo group, a further improvement was seen in general health domain at week 48 compared to week $30(+2.4 ; F=7.063, P=0.010)$ (Figure 3b).

The maximum improvement in mental health composite scores was seen at week 30 with no further improvement at week 48 , while physical health composite scores improved significantly at week 30 and continued to improve further at week $48(P<0.05)$ (Figure 4).
After adjusting for baseline differences, the covariates of body mass index, systolic blood pressure, diastolic blood pressure and total AMS scores, using multivariate methods, only three domains of QoL in SF12 remained significant. They were the vitality, social functioning, and mental health composite scores (Table 2).

\section{Changes in IPSS, clinical assessments and biochemistry safety profiles}

IPSS scores did not change significantly in both active treatment and placebo groups. The mean IPSS scores for active treatment and placebo groups were $10.2 \pm 7.2$ and $11.1 \pm 8.4$ at baseline, and $7.8 \pm 6.4$ and $9.5 \pm 8.2$ at 48 weeks $(F=0.462, P=0.609)$, respectively. Similarly, weight, height, systolic blood pressure, diastolic blood pressure and prostate size from digital rectal examination did not change significantly after interventions (data not shown). However, the haematocrit level, serum bilirubin and serum PSA increased significantly $(P<0.01)$ in the active treatment group compared to placebo (Table 3). The mean levels were still within normal limits.

\section{DISCUSSION}

In this double-blind randomized controlled trial, we found that prior to adjusement for baseline differences, the administration of deep intramuscular injection of $1000 \mathrm{mg}$ of TU had resulted in significant improvement in the physical and mental health composite scores and the five domains of QoL of SF-12. More improvement was seen in vitality, followed by general health $(+6.1)$, social $(+5.7)$, physical role $(+3.0)$ and emotional role functioning $(+1.8)$. The improvement in mental health composite scores was seen earlier than the physical composite scores, reaching its peak at 30 weeks after the treatment, while the improvement in physical health composite scores continued beyond 30 weeks of treatment. It is important to note that men

Table 2 Comparing the SF-12 domain scores for quality of life (QoL) between placebo and active treatment arms over time

\begin{tabular}{|c|c|c|c|c|c|c|c|c|c|c|c|c|}
\hline \multirow[t]{2}{*}{ QoL domains } & & \multirow[t]{2}{*}{ Baseline } & \multirow[t]{2}{*}{ Week 30} & \multirow[t]{2}{*}{ Week 48} & \multicolumn{2}{|c|}{$\begin{array}{c}\text { ANOVA (comparing scores } \\
\text { within the group from } \\
\text { baseline) }\end{array}$} & \multicolumn{3}{|c|}{ Repeated measure ANOVA* } & \multicolumn{3}{|c|}{$\begin{array}{l}\text { Adjusted repeated measure } \\
\qquad \text { ANOVA** }^{*}\end{array}$} \\
\hline & & & & & $\mathrm{F}$ & $\mathrm{P}$ & $\mathrm{F}$ & $\mathrm{P}$ & $\begin{array}{l}\text { Observed } \\
\text { power }\end{array}$ & $\mathrm{F}$ & $\mathrm{P}$ & $\begin{array}{c}\text { Observed } \\
\text { power }\end{array}$ \\
\hline \multirow[t]{2}{*}{ Physical functioning } & $P$ & 48.0 & 47.6 & 48.5 & 0.377 & 0.686 & 2.155 & 0.122 & 0.421 & 1.443 & 0.239 & 0.356 \\
\hline & $A$ & 45.0 & 46.7 & 49.0 & 5.057 & 0.013 & & & & & & \\
\hline \multirow{2}{*}{$\begin{array}{l}\text { Role functioning } \\
\text { (physical) }\end{array}$} & $P$ & 26.7 & 26.5 & 27.2 & 0.835 & 0.424 & 4.142 & 0.021 & 0.695 & 4.288 & 0.015 & 0.711 \\
\hline & $A$ & 25.5 & 27.3 & 28.5 & 11.491 & 0.000 & & & & & & \\
\hline \multirow[t]{2}{*}{ Bodily pain } & $P$ & 45.9 & 45.6 & 46.7 & 0.318 & 0.728 & 2.112 & 0.124 & 0.373 & 0.937 & 0.376 & 0.211 \\
\hline & $A$ & 45.7 & 49.0 & 49.4 & 4.602 & 0.015 & & & & & & \\
\hline \multirow[t]{2}{*}{ General health } & $P$ & 41.2 & 41.0 & 43.5 & 4.436 & 0.014 & 4.023 & 0.022 & 0.686 & 3.079 & 0.053 & 0.561 \\
\hline & $A$ & 39.8 & 43.5 & 45.9 & 14.261 & 0.000 & & & & & & \\
\hline \multirow[t]{2}{*}{ Vitality } & $P$ & 47.2 & 49.1 & 48.1 & 0.936 & 0.383 & 6.972 & 0.002 & 0.909 & 6.923 & 0.002 & 0.904 \\
\hline & $A$ & 44.9 & 51.0 & 51.9 & 23.950 & 0.000 & & & & & & \\
\hline \multirow[t]{2}{*}{ Social functioning } & $P$ & 44.8 & 46.3 & 45.4 & 0.631 & 0.534 & 3.198 & 0.048 & 0.576 & 3.940 & 0.025 & 0.670 \\
\hline & $A$ & 42.6 & 47.5 & 48.3 & 10.093 & 0.000 & & & & & & \\
\hline \multirow{2}{*}{$\begin{array}{l}\text { Role functioning } \\
\text { (emotional) }\end{array}$} & $P$ & 19.4 & 19.6 & 19.1 & 0.392 & 0.622 & 3.448 & 0.047 & 0.556 & 2.670 & 0.088 & 0.450 \\
\hline & $A$ & 19.0 & 21.0 & 20.8 & 7.092 & 0.004 & & & & & & \\
\hline \multirow[t]{2}{*}{ Mental health } & $P$ & 47.9 & 48.8 & 50.3 & 1.785 & 0.172 & 2.882 & 0.063 & 0.536 & 2.334 & 0.104 & 0.449 \\
\hline & $A$ & 47.3 & 52.2 & 52.4 & 11.571 & 0.000 & & & & & & \\
\hline \multirow{2}{*}{$\begin{array}{l}\text { Physical health } \\
\text { composite scores }\end{array}$} & $P$ & 43.7 & 43.1 & 44.5 & 1.311 & 0.273 & 3.652 & 0.027 & 0.659 & 2.542 & 0.084 & 0.493 \\
\hline & $A$ & 41.9 & 44.0 & 45.9 & 9.363 & 0.000 & & & & & & \\
\hline \multirow{2}{*}{$\begin{array}{l}\text { Mental health } \\
\text { composite scores }\end{array}$} & $P$ & 37.6 & 38.9 & 38.6 & 1.392 & 0.253 & 4.514 & 0.018 & 0.704 & 4.860 & 0.013 & 0.740 \\
\hline & $A$ & 37.1 & 41.8 & 41.5 & 15.991 & 0.000 & & & & & & \\
\hline
\end{tabular}

Abbreviations: $\mathrm{A}$, active treatment; $\mathrm{P}$, placebo.

*Comparing between two intervention groups over time by including the interaction term of 'intervention $\times$ time'.

**Adjusted for baseline BMI, SBP, DBP and total AMS scores differences. 


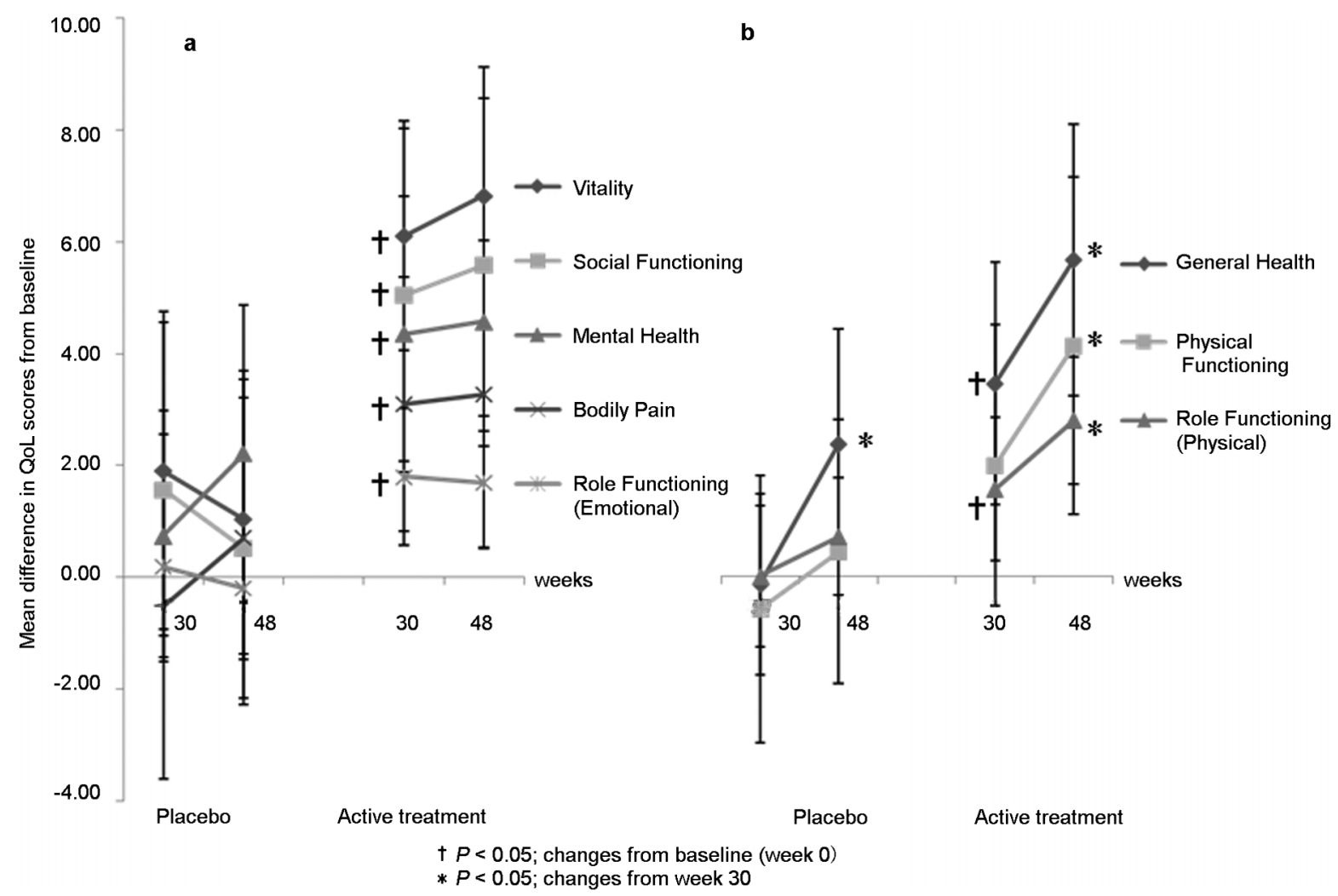

Figure 3 Changes from baseline score in the eight domains of SF-12. QoL, quality of life.

enrolled in this study had borderline low serum TT, although the mean total and subscale AMS scores were in the moderate to severe categories. ${ }^{30}$ Despite vigorous randomisation as described, baseline differences were still observed. After adjusting for these potential confounders by using multivariate analysis, the significant improvement between active treatment and placebo group was observed in three domains of QoL scores, which were the vitality, social functioning and role functioning (physical), and mental health composite scores. In these components, the observed power of the statistics is considerable compared to those in domains with insignificant improvement after the adjustment (Table 2).

Serum TT was measured at weeks 18 and 48 after the first injection. In the active treatment group, we noted that the serum TT was higher

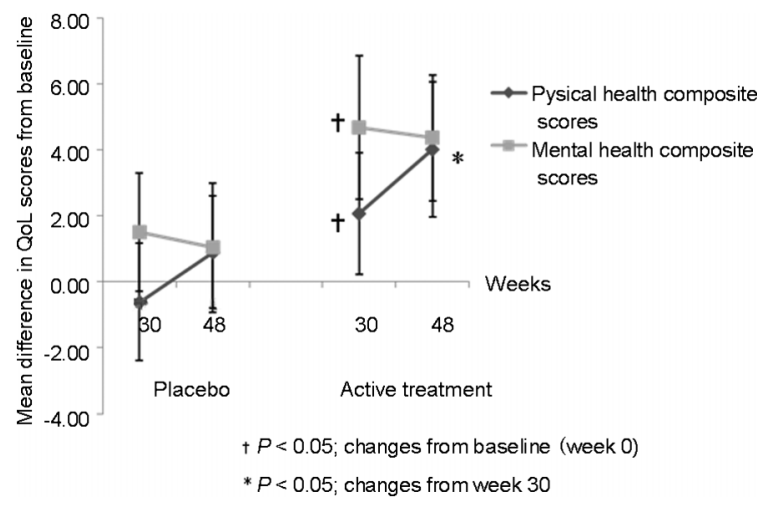

Figure 4 Changes from baseline (with 95\% Cl) in the composite scores of SF-12. QoL, quality of life; SF-12, Short-Form-12. at week 48 compared to week 18. This could be due to the time difference between the injection and serum TT assessment. The serum TT assessments were done at trough level at week 18 (just before the third injection) and at 6 weeks after the final injection. As expected, serum TT would not have settled to its trough level after the final injection. Other randomized trials using the same dosing intervals as our study showed stabilized trough level within the normal ranged of serum TT at week $18 .^{31,32}$ Hence, it is likely that the trough level measured at week 18 in our study represents the stable trough level and the higher level measured at week 48 represent is not an increasing trend of serum TT, but the peak of the final TU dosing. Although this level is within physiological limits, for safety issue in long-term treatment after 1 year, regular monitoring of serum TT is warranted during and after 1 year of treatment to avoid over-replacement of testosterone. In placebo group, serum TT was noted to increase slightly from baseline. However, their serum TT was still below $12 \mathrm{nmol}^{-1}$. Therefore, they were still in hypogonadal state. The slight increase may represent a regression to the mean due to selection pressure from two 'low' pre-treatment baseline blood samples. This raises the possibility of a need for more than two pre-treatment baselines in future studies.

In this study, one participant (in the placebo group) was on rosiglitazone and 18 (10 in the placebo group and 8 in active treatment group) were on statins therapy prior to the study. This may affect their level of serum TT because rosiglitazone has been associated with elevated serum $\mathrm{TT}^{33}$ and statins have been associated with lower serum TT. ${ }^{34}$ However, the level of serum TT for the participant on rosiglitazone in this study remained at $10.4 \mathrm{nmol} \mathrm{l}^{-1}$ at week 48 . For participants on statins, the serum TT, as expected, increased in the active treatment group or did not change significantly in the placebo group at week 48. In the active treatment group, their serum TT increased 
Table 3 Biochemical parameters with significant increases between placebo and active treatment arm (Nebido)

\begin{tabular}{|c|c|c|c|c|c|c|}
\hline \multirow[t]{3}{*}{ Variables } & \multicolumn{2}{|c|}{ Placebo } & \multicolumn{2}{|c|}{ Nebido } & \multirow{3}{*}{$\mathrm{F}$} & \multirow{3}{*}{$P$} \\
\hline & Baseline & 48 weeks & Baseline & 48 weeks & & \\
\hline & Mean \pm s.d. & Mean $\pm s . d$ & Mean \pm s.d. & Mean \pm s.d. & & \\
\hline PSA (ng ml ${ }^{-1}$ ) & $0.9 \pm 0.8$ & $1.1 \pm 1.0$ & $0.8 \pm 0.6$ & $1.2 \pm 1.0$ & 4.789 & 0.011 \\
\hline Haematocrit (\%) & $43.4 \pm 2.8$ & $43.2 \pm 3.1$ & $43.1 \pm 3.4$ & $48.7 \pm 4.4$ & 53.149 & $<0.001$ \\
\hline Total bilirubin $\left(\mu \mathrm{mol} \mathrm{I}^{-1}\right)$ & $11.1 \pm 4.4$ & $11.8 \pm 4.8$ & $12.3 \pm 5.8$ & $15.6 \pm 7.0$ & 12.295 & 0.001 \\
\hline
\end{tabular}

Abbreviation: PSA, prostate-specific antigen.

from 7.4 to $18.3 \mathrm{nmoll}^{-1}$ compared to baseline, whereas in the placebo group, the serum TT changed by $-0.6-5.2 \mathrm{nmol}^{-1}$ from baseline. Therefore, concurrent rosiglitazone and statins therapy in this study did not result in alteration of serum TT.

The impact of testosterone therapy on QoL has been studied since 1999. ${ }^{19,35,36}$ However, many studies have shown conflicting results in the generic QoL scales, ${ }^{19,36}$ compared to studies using disease-specific QoL scales, which often showed positive results. ${ }^{19}$ The conflicting results are mainly due to small sample size $e^{36-42}$ and recruiting men solely based on serum testosterone level. ${ }^{35,38,42-45}$ Haren et al. ${ }^{40}$ recruited men with TDS and showed no improvement in QoL with oral TU, which has considerable disadvantage in efficacy compared to injectable TU. ${ }^{46}$ It is difficult to conclude with confidence as the generic QoL scale used was based on one statement on how patient would rate their QoL in a 1-10 visual analogue scale. Kaniguchi et al. ${ }^{47}$ showed a positive effect of intramuscular testosterone enanthate on the QoL in men with TDS using generic QoL scale (SF-36). However, the effect size was likely to be overestimated as it was a cohort study based on self-returning questionnaire among patients who had undergone testosterone replacement therapy. Our study used double-blind randomized controlled trial design which was more rigorous in design in providing the evidence on the effectiveness of testosterone replacement therapy on generic QoL.

A qualitative study has shown that TDS adversely affects the energy level, emotion as well as social, mental, physical and sexual functioning, ${ }^{11}$ whereas a quantitative survey has revealed that TDS affects the memory, energy, sex life and physical stamina. ${ }^{48}$ We found that testosterone therapy with injectable TU improved mental health, in particular, vitality (reflects the energy level), social functioning and physical role functioning. Although physical health composite score did not show statistically significant improvement after adjusting for basement differences, there was an improving trend shown as early as week 30 that continued to week 48 suggesting a continuous improvement in physical strength. This is in contrast to other study which showed an improvement in grip strength 90 weeks after the injection of TU, but not at 30 weeks. ${ }^{49}$ However, our findings are more consistent with a recent review by Saad et al., ${ }^{7}$ where physical strength was noted to improve after 3 months and peak at 12 months after initiation of testosterone replacement therapy. Our study also showed an earlier improvement in mental health composite scores at week 30 and remained at the same level throughout the rest of the study period. This finding is also consistent with the review by Saad et al., ${ }^{7}$ where most of the positive effects seen in the psychological status were within 30 weeks of treatment. However, most of the psychological variables reviewed by Saad et al. ${ }^{7}$ were related to depression and disease specific QoL scales. Previous studies have shown beneficial effects of oral TU on men with TDS using disease-specific QoL scales. ${ }^{50,51}$ However, studies using generic SF-36 QoL scales that recruited men based on the criterion of low serum testosterone level alone had either shown improvement in the physical components of QoL scales ${ }^{35,44}$ or no effect in any of the components. ${ }^{38,42,43,45}$ The lack of improvement seen was because the participants in these studies might not have TDS, as having a low serum TT alone does not fulfill the diagnosis of TDS. Therefore, for patients with TDS, testosterone replacement therapy is likely to have an improvement in their QoL as measured by generic QoL scales.

The mean baseline physical and mental composite scores for men in our study were $42.8 \pm 7.1$ and $37.1 \pm 8.5$, respectively, which was lower than the mean SF-36 scores in our general population that ranged from 52.0 to 86.3 points, ${ }^{52}$ This suggest that men with TDS have poorer QoL. Although their QoL improved with injectable testosterone after 48 weeks of treatment, it was still below the average of the Malaysian general population. The improvement in our study was smaller compared to two other randomized controlled trials using generic QoL scales. However, the findings might not be comparable because Snyder et al. ${ }^{35}$ and Nieschlag et al. ${ }^{46}$ recruited men with just one criterion of low serum testosterone level and their baseline QoL scores were higher, ranged from 54 to $>90$ points. ${ }^{35,46}$

No significant adverse event was observed in this study. The incidence rates of cardiac events for treatment and placebo groups were $3.6 \%$ and $3.5 \%$, respectively. These incidence rates are higher than the national estimate of acute coronary event incidence rate, which was $141 / 100000$ population. ${ }^{53}$ Thus, the participants in our study were from higher cardiovascular risk category than the general population. There was no excess cases of cardiovascular events in the active treatment group compared to the placebo and this was consistent with the results of a meta-analysis by Haddad et al. ${ }^{54}$ Nevertheless, a recent study has shown significant higher risk of cardiac events in the elderly with limited mobility receiving testosterone gel therapy. ${ }^{55}$ Needless to say, the present study highlights that adverse coronary events may be associated with testosterone. Further study is needed to evaluate this. The significant increase in serum PSA and haematocrit level is expected as their association with testosterone treatment has been well documented. ${ }^{17,18,56}$ However, the increase in the levels of PSA and haematocrit was still within normal limits. Six participants had PSA levels exceeded $4 \mathrm{ng} \mathrm{ml}^{-1}$ over the trial period with the highest level at $5.9 \mathrm{ng} \mathrm{ml}^{-1}$, but the PSA levels declined at week 48 in three of them. The other three participants had the levels of 5.3, 5.1 and $4.8 \mathrm{ng} \mathrm{ml}^{-1}$ respectively at week 48 . On follow-up, the levels returned to their pretrial levels. The significant increased in serum total bilirubin level in active treatment group has been reported in the trial involving TU injection, but the increased was small. ${ }^{31}$ Although the increased level of bilirubin was still within the normal range, the small increase in serum total bilirubin may be significant for patients with underlying liver disease. Hence, besides monitoring for serum PSA level and haematocrit, ${ }^{2}$ further study may be needed to confirm the effect of TU on serum total bilirubin level.

QoL score in SF-12 was a secondary outcome in this project. Although this study was underpowered to detect changes in the QoL 
scores, except for the vitality domain (Table 2), three domains of the QoL showed a significant difference between active treatment and placebo after adjusting for baseline differences. The insignificant difference in the other five domains might be due to insufficient power of the study. Nevertheless, the mental composite scores showed significant improvement, and it is possible that all domains of the QoL would improve with TU injection with a longer duration of follow-up or a larger sample size. Although positive effects of TU injection on QoL were demonstrated, these may not translate into improvement in the participants' satisfaction with testosterone therapy and in their desire to continue with the treatment. Future studies should explore patients' satisfaction and their desire for long-term treatment.

\section{CONCLUSION}

This randomized controlled trial has demonstrated the effectiveness of long-acting deep intramuscular injection of TU in improving the mental health component of QoL in men with TDS. It has also shown that men with TDS who have a borderline low serum testosterone and are symptomatic would benefit from the treatment. While the improvement in SF-12 composite scores was apparent within 30 weeks of treatment, it is important to note that the physical composite scores continued to improve at 48 weeks of treatment. Therefore, testosterone treatment may be indicated in men who have a poor quality of life from TDS.

\section{COMPETING FINANCIAL INTERESTS}

This study was fully supported by Bayer Schering Pharma. Otherwise, we have no competing interests. The sponsor was not involved in the design, conduct, analysis of the study nor writing of this manuscript.

\section{AUTHOR CONTRIBUTIONS}

SFT participated in designing the study, analysed the results and drafted the manuscript. CJN participated in designing the study, and helped to analyse the results and draft the manuscript. EMK participated in designing and conducting the study and helped to draft the manuscript. VKML, BCL and EGL participated in designing and conducting the study. HMT conceived, designed and coordinated the study. All authors have read and approved the final manuscript.

\section{ACKNOWLEDGMENTS}

We would like to thank the Medical Ethics Committee, University of Malaya Medical Centre for the ethics approval (approval number: 631.11) and Bayer Schering Pharma for funding the whole project. We also would like to thank Associate Professor Karuthan Chinna for his assistant in statistical analysis and our research assistant, Miss Chocho Yi.

1 Bassil N, Morley JE. Late-life onset hypogonadism: a review. Clin Geriatr Med 2010; 26: 197-222.

2 Wang C, Nieschlag E, Swerdloff R, Behre HM, Hellstrom WJ et al. Investigation, treatment, and monitoring of late-onset hypogonadism in males: ISA, ISSAM, EAU, EAA, and ASA recommendations. J Androl 2009; 30: 1-9.

3 Wang $\mathrm{C}$, Jackson G, Jones TH, Matsumoto AM, Nehra A et al. Low testosterone associated with obesity and the metabolic syndrome contributes to sexual dysfunction and cardiovascular disease risk in men with type 2 diabetes. Diabetes Care 2011; 34: 1669-75.

4 Kazi M, Geraci SA, Koch CA. Considerations for the diagnosis and treatment of testosterone deficiency in elderly men. Am J Med 2007; 120: 835-40.

5 Ullah MI, Washington T, Kazi M, Tamanna S, Koch CA. Testosterone deficiency as a risk factor for cardiovascular disease. Horm Metab Res 2011; 43: 153-64.

6 Traish AM, Miner MM, Morgentaler A, Zitzmann M. Testosterone deficiency. Am J Med 2011; 124: 578-87.

7 Saad F, Aversa A, Isidori AM, Zafalon L, Zitzmann M et al. Onset of effects of testosterone treatment and time span until maximum effects are achieved. Eur $J$ Endocrinol 2011; 165: 675-85.
8 Araujo AB, Esche GR, Kupelian V, O'Donnell AB, Travison TG et al. Prevalence of symptomatic androgen deficiency in men. J Clin Endocrinol Metab 2007; 92: 4241-7.

9 Khoo EM, Tan HM, Low WY. Erectile dysfunction and comorbidities in aging men: an urban cross-sectional study in Malaysia. J Sex Med 2008; 5: 2925-34.

10 Lunenfeld B, Saad F, HoesI CE. ISA, ISSAM and EAU recommendations for the investigation, treatment and monitoring of late-onset hypogonadism in males: scientific background and rationale. Aging Male 2005; 8: 59-74.

11 Novak A, Brod M, Elbers J. Andropause and quality of life: findings from patient focus groups and clinical experts. Maturitas 2002; 43: 231-7.

12 Moncada I. Testosterone and men's quality of life. Aging Male 2006; 9: 189-93.

13 Maggi M, Schulman C, Quinton R, Langham S, Uhl-Hochgraeber K. The burden of testosterone deficiency syndrome in adult men: economic and quality-of-life impact. J Sex Med 2007; 4: 1056-69.

14 Low WY, Tong SF, Tan HM. Erectile dysfunction, premature ejaculation and hypogonadism and men's quality of life: an Asian perspective. J Men'Health 2008; 5: $282-8$.

15 Yasuda M, Furuya K, Yoshii T, Ide H, Muto S et al. Low testosterone level of middleaged Japanese men-the association between low testosterone levels and quality-oflife. J Men'Health Gend 2007; 4: 149-55.

16 Hwang TI, Lo HC, Tsai TF, Chiou HY, Hwang TI. Association among hypogonadism, quality of life and erectile dysfunction in middle-aged and aged male in Taiwan. Int J Impot Res 2007; 19: 69-75.

17 Edelstein D, Basaria S. Testosterone undecanoate in the treatment of male hypogonadism. Expert Opin Pharmacother 2010; 11: 2095-106.

18 Saad F, Kamischke A, Yassin A, Zitzmann M, Schubert M et al. More than eight years' hands-on experience with the novel long-acting parenteral testosterone undecanoate. Asian J Androl 2007; 9: 291-7.

19 Langham S, Maggi M, Schulman C, Quinton R, Uhl-Hochgraeber K. Health-related quality of life instruments in studies of adult men with testosterone deficiency syndrome: a critical assessment. J Sex Med 2008; 5: 2842-52.

20 Ho CC, Tong SF, Low WY, Ng CJ, Khoo EM et al. A randomized double blind placebocontrolled trial on the effect of long-acting testosterone treatment on the Aging Male Symptoms scale. BJU Int; e-pub ahead of print 17 November 2011; doi: 10.1111/ j.1464-410X.2011.10755.x.

$21 \mathrm{Ng}$ CJ, Tan HM, Khoo EM, Low WY. Under-detection and under treatment of common men's health problems. Asian J Androl 2007; 9: 418.

22 Morley JE, Perry HM III, Kevorkian RT, Patrick P. Comparison of screening questionnaires for the diagnosis of hypogonadism. Maturitas 2006; 53: 424-9.

23 Tan HM, Khoo EM, Ng CJ, Low WY, Yap PK et al. The aging males' symptoms' scale (AMS) - A general screening instrument for aging males' health problem? Eur Urol Suppl 2007; 6: 107(Abstract).

24 Sararaks S, Azman AB, Low LL, Rugayah B, Aziah AM et al. Validity and reliability of the SF-36: the Malaysian context. Med J Malaysia 2005; 60: 163-79.

25 Ware J, Jr., Kosinski M, Keller SD. A 12-Item Short-Form Health Survey: construction of scales and preliminary tests of reliability and validity. Med Care 1996; 34: 220-33.

26 Jenkinson C, Layte R. Development and testing of the UK SF-12 (short form health survey). J Health Serv Res Policy 1997; 2: 14-8.

27 Jenkinson C, Layte R, Jenkinson D, Lawrence K, Petersen S et al. A shorter form health survey: can the SF-12 replicate results from the SF-36 in longitudinal studies? J Public Health Med 1997; 19: 179-86.

28 Lam CL, Tse EY, Gandek B, Lam CL, Tse EY et al. Is the standard SF-12 health survey valid and equivalent for a Chinese population? Qual Life Res 2005; 14: 539-47.

29 Pu YS, Chiang HS, Lin CC, Huang CY, Huang KH et al. Changing trends of prostate cancer in Asia. Aging Male 2004; 7: 120-32.

30 Heinemann LA, Zimmermann T, Vermeulent A, Thiel C, Hummel W. A new 'aging males' symptoms rating scale. Aging Male 1999; 2: 105-14.

31 Kalinchenko SY, Tishova YA, Mskhalaya GJ, Gooren LJ, Giltay EJ et al. Effects of testosterone supplementation on markers of the metabolic syndrome and inflammation in hypogonadal men with the metabolic syndrome: the double-blinded placebo-controlled Moscow study. Clin Endocrinol (Oxf) 2011; 73: 602-12.

32 Fennell C, Sartorius G, Ly LP, Turner L, Liu PY et al. Randomized cross-over clinical trial of injectable vs. implantable depot testosterone for maintenance of testosterone replacement therapy in androgen deficient men. Clin Endocrinol (Oxf) 2010; 73: 102-9.

33 Kapoor D, Channer KS, Jones TH. Rosiglitazone increases bioactive testosterone and reduces waist circumference in hypogonadal men with type 2 diabetes. Diab Vasc Dis Res 2008; 5: 135-7.

34 Stanworth RD, Kapoor D, Channer KS, Jones TH. Statin therapy is associated with lower total but not bioavailable or free testosterone in men with type 2 diabetes. Diabetes Care 2009; 32: 541-6.

35 Snyder PJ, Peachey H, Hannoush P, Berlin JA, Loh L et al. Effect of testosterone treatment on body composition and muscle strength in men over 65 years of age. J Clin Endocrinol Metab 1999; 84: 2647-53.

36 Haren MT, Kim MJ, Tariq SH, Wittert GA, Morley JE. Andropause: a quality-of-life issue in older males. Med Clin North Am 2006; 90: 1005-23.

37 English KM, Steeds RP, Jones TH, Diver MJ, Channer KS. Low-dose transdermal testosterone therapy improves angina threshold in men with chronic stable angina: a randomized, double-blind, placebo-controlled study. Circulation 2000; 102: 190611

38 Reddy P, White CM, Dunn AB, Moyna NM, Thompson PD. The effect of testosterone on health-related quality of life in elderly males-a pilot study. J Clin Pharm Ther 2000; 25: 421-6. 
39 Park NC, Yan BQ, Chung JM, Lee KM. Oral testosterone undecanoate (Andriol) supplement therapy improves the quality of life for men with testosterone deficiency. Aging Male 2003; 6: 86-93.

40 Haren MT, Wittert GA, Chapman IM, Coates P, Morley JE. Effect of oral testosterone undecanoate on visuospatial cognition, mood and quality of life in elderly men with low-normal gonadal status. Maturitas 2005; 50: 124-33.

41 Malkin CJ, Pugh PJ, West JN, van Beek EJ, Jones TH et al. Testosterone therapy in men with moderate severity heart failure: a double-blind randomized placebo controlled trial. Eur Heart J 2006; 27: 57-64.

42 Ly LP, Jimenez M, Zhuang TN, Celermajer DS, Conway AJ et al. A double-blind, placebo-controlled, randomized clinical trial of transdermal dihydrotestosterone gel on muscular strength, mobility, and quality of life in older men with partial androgen deficiency. J Clin Endocrinol Metab 2001; 86: 4078-88.

43 Kenny AM, Bellantonio S, Gruman CA, Acosta RD, Prestwood KM. Effects of transdermal testosterone on cognitive function and health perception in older men with low bioavailable testosterone levels. J Gerontol A Biol Sci Med Sci 2002; 57 : M460-5.

44 Katznelson L, Robinson MW, Coyle CL, Lee H, Farrell CE. Effects of modest testosterone supplementation and exercise for 12 weeks on body composition and quality of life in elderly men. Eur J Endocrinol 2006; 155: 867-75.

45 Emmelot-Vonk MH, Verhaar HJ, Nakhai Pour HR, Aleman A, Lock TM et al. Effect of testosterone supplementation on functional mobility, cognition, and other parameters in older men: a randomized controlled trial. JAMA 2008; 299: 3952.

46 Nieschlag E, Behre H, Bouchard P, Corrales J, Jones $T$ et al. Testosterone replacement therapy: current trends and future directions. Hum Reprod Update 2004; 10: 409.
47 Taniguchi H, Kawa G, Kinoshita H, Matsuda T. Androgen replacement therapy improves health-related quality of life in late onset hypogonadism patients. Hinyokika Kiyo - Acta Urologica Japonica 2009; 55 (12): 741-4.

48 McMillan CV, Bradley C, Giannoulis M, Martin F, Sonksen PH. Preliminary development of a new individualised questionnaire measuring quality of life in older men with agerelated hormonal decline: the A-RHDQoL. Health Qual Life Outcomes 2003; 1: 51.

49 Minnemann T, Schubert M, Freude S, Hubler D, Gouni-Berthold I et al. Comparison of a new long-acting testosterone undecanoate formulation vs testosterone enanthate for intramuscular androgen therapy in male hypogonadism. J Endocrinol Invest 2008, 31: 718-23

50 Heinemann LA, Moore C, Dinger JC, Stoehr D. Sensitivity as outcome measure of androgen replacement: the AMS scale. Health Qual Life Outcomes 2006; 4: 23.

51 Haren M, Chapman I, Coates P, Morley J, Wittert G. Effect of 12 month oral testosterone on testosterone deficiency symptoms in symptomatic elderly males with low-normal gonadal status. Age Ageing 2005; 34: 125-30.

52 Azman AB, Sararaks S, Rugayah B, Low LL, Azian AA et al. Quality of life of the Malaysian general population: results from a postal survey using the SF-36. Med $J$ Malaysia 2003; 58: 694-711.

53 Wan Ahmad WA, Sim KH. Annual Report of the NCVD-ACS Registry Malaysia 2006 National Cardiovascular Disease Database, Kuala LumpurMalaysia, 2006.

54 Haddad RM, Kennedy CC, Caples SM, Tracz MJ, Bolona ER et al. Testosterone and cardiovascular risk in men: a systematic review and meta-analysis of randomized placebo-controlled trials. Mayo Clin Proc 2007; 82: 29-39.

55 Basaria S, Coviello AD, Travison TG, Storer TW, Farwell WR et al. Adverse events associated with testosterone administration. N Engl J Med 2010; 363: 109-22.

56 von Eckardstein S, Nieschlag E. Treatment of male hypogonadism with testosterone undecanoate injected at extended intervals of 12 weeks: a phase II study. J Androl 2002; 23: 419-25. 